Published in final edited form as:

Crit Care Med. 2016 August ; 44(8): e614-e624. doi:10.1097/CCM.0000000000001606.

\title{
Molecular mechanisms autonomic dysfunction and impaired cardiac contractility in critical illness
}

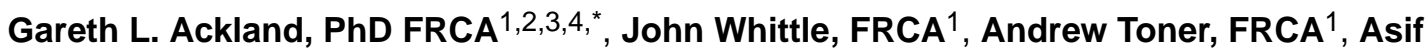 \\ Machhada, BSc ${ }^{3}$, Ana Gutierrez Del Arroyo, $\mathrm{PhD}^{1,2}$, Alberto Sciuso, MD ${ }^{1}$, Nicholas Jenkins, \\ FRCA $^{1}$, Alex Dyson, PhD ${ }^{1}$, Richard Struthers, FRCA ${ }^{5}$, Robert Sneyd, PhD FRCA ${ }^{5}$, Gary \\ Minto, FRCA ${ }^{5}$, Mervyn Singer, FRCP ${ }^{1,2}$, Ajay M. Shah, PhD FRCP ${ }^{6}$, and Alexander V. \\ Gourine, $\mathrm{PhD}^{3}$ \\ ${ }^{1}$ Clinical Physiology, Division of Medicine, University College London, London, UK \\ 2University College London/University College London Hospitals NIHR Biomedical Research \\ Centre, London, UK \\ ${ }^{3}$ Centre for Cardiovascular and Metabolic Neuroscience, Neuroscience, Physiology and \\ Pharmacology, University College London, UK \\ ${ }^{4}$ William Harvey Research Institute, Queen Mary University of London, Charterhouse Square, \\ London EC1M 6BQ, UK \\ ${ }^{5}$ Peninsula Medical School, Plymouth, UK \\ ${ }^{6}$ British Heart Foundation Centre for Excellence, Kings College, London, UK
}

\section{Abstract}

Copyright form disclosures:Dr. Ackland received support for travel from Baxter healthcare (talk on beta-blockers 2010); has a patent through UCL Business (patent filed related to assessment of autonomic function - patent application 1414161.8); and received support for article research from the BRITISH HEART FOUNDATION, Wellcome Trust, and Research Councils UK (RCUK). His institution received grant support from the Academy Medical Sciences/Health Foundation Clinician Scientist Award (GLA) (GLA and AVG are supported by the British Heart Foundation Programme Grant. Part of this work was undertaken at UCLH/UCL who received a proportion of funding from the UK Department of Health's NIHR Biomedical Research Centres funding scheme). Dr. Toner's institution has a patent through UCL Business (patent filed related to assessment of autonomic function - patent application 1414161.8). Dr. Whittle has a patent through UCL Business (patent filed related to assessment of autonomic function - patent application 1414161.8). Dr. Machhada received support for article research from the Research Councils UK (RCUK). Dr. Dyson served as a board member for Magnus Oxygen, consulted for Magnus Oxygen; is employed by Magnus Life Science/University College London, has a patent related to Magnus Oxygen and not the current manuscript, and has shares in Magnus Oxygen. Dr. Struthers' institution received grant support from the BJA/RCOA Project grant. Dr. Minto's institution received grant support from BJA/RCOA Project grant. Dr. Shah received support for article research from the BRITISH HEART FOUNDATION. His institution received grant support from King's College London BHF Centre of Excellence. Dr. Gourine received support for article research from the NIH, Research Councils UK (RCUK), and the British Heart Foundation. His institution received grant support from the Wellcome Trust Senior Research Fellow and British Heart Foundation Programme Grant. The remaining authors have disclosed that they do not have any potential conflicts of interest.

"Corresponding author: Gareth Ackland PhD FRCA FFICM, William Harvey Research Institute, Queen Mary University of London John Vane Building, Charterhouse Square, London EC1M 6BQ, E-mail: g.ackland@qmul.ac.uk Fax: +44 20 7679 6683; Tel: +44 20 76796683.

Author Contributions: Conceived and designed the experiments: GLA. Performed the experiments: GLA, JW, AGDA, AM. AT, AD, SR, PS, JRS, RS, GM, AVG. Analyzed the data: GLA, JW, AGDA, AM, AT, AD, GM, AVG, AMS, NJ. Contributed reagents/ materials/analysis tools: GLA, RS, JRS, GM, MS, AVG, AS. Wrote the first draft of the manuscript: GLA, AVG. Contributed to the writing of the manuscript: GLA, JW, AGDA, AM, AT, AD, JRS, GM, MS, AVG, AMS. ICMJE criteria for authorship read and met: GLA, JW, AGDA, AT, AM, AD, SR, PS, JRS, RS, GM, MS, AVG, AMS, NJ. Agree with manuscript results and conclusions: GLA, JW, AGDA, AT, AM, AD, SR, PS, JRS, RS, GM, MS, AVG, AMS, NJ. 
Objectives: Molecular mechanisms linking autonomic dysfunction with poorer clinical outcomes in critical illness remain unclear. We hypothesized that baroreflex dysfunction alone is sufficient to cause cardiac impairment through neurohormonal activation of (NADPH oxidase-dependent) oxidative stress resulting in increased expression of G-protein coupled receptor kinase (GRK)-2, a key negative regulator of cardiac function.

Design: Laboratory/clinical investigations.

Setting: University laboratory/medical centers.

Subjects: Adult rats; wild-type/NAPDH oxidase subunit-2 (NOX-2) deficient mice; elective surgical patients.

Interventions: Cardiac performance was assessed by transthoracic echocardiography following experimental baroreflex dysfunction (BD, sino-aortic denervation) in rats and mice. Immunoblots assessed GPCR recycling proteins expression in rodent cardiomyocytes and patient mononuclear leukocytes. In surgical patients, heart rate recovery after cardio-pulmonary exercise testing, time/ frequency measures of parasympathetic parameters were related to the presence/absence of BD (defined by spontaneous baroreflex sensitivity of $<6 \mathrm{~ms} \mathrm{mmHg}^{-1}$ ). The associations of BD with intraoperative cardiac function and outcomes were assessed.

Measurements and Main Results: Experimental BD in rats and mice resulted in impaired cardiac contractility and upregulation of GRK-2 expression. In mice, genetic deficiency of gp91 NADPHoxidase (NOX-2) subunit prevented upregulation of GRK-2 expression in conditions of BD and preserved cardiac function. BD was present in 81/249 (32.5\%) patients, and was characterized by lower parasympathetic tone and increased GRK-2 expression in mononuclear leukocytes. BD in patients was also associated with impaired intraoperative cardiac contractility. Critical illness and mortality were more frequent in surgical patients with BD (relative risk: 1.66 [95\%CI:1.16-2.39]; $\mathrm{p}=0.006)$.

Conclusions: Reduced baroreflex sensitivity is associated with NOX-2 mediated upregulation of GRK-2 expression in cardiomyocytes and impaired cardiac contractility. Autonomic dysfunction predisposes patients to the development of critical illness and increases mortality.

\section{Keywords}

autonomic dysfunction; multiple organ dysfunction syndrome; cardiac contractility; Sepsis; GPCR; GRK

\section{Introduction}

Maintenance of biologic variability is strongly associated with health and preservation of organ function. (1) Physiologic variability is modulated by the autonomic nervous system, perturbations of which occur at the onset of pathophysiologic insults resulting in critical illness regardless of etiology. Several features of established critical illness are compatible with a direct mechanistic role for autonomic dysfunction in causing multi-organ dysfunction, although patient data exploring this hypothesis remain limited. Prolonged bed rest (even in healthy volunteers) induces rapid detrimental changes in baroreflex autonomic control, (2) 
which is associated with excess morbidity and mortality during critical illness. (3) A key feature of experimental baroreflex dysfunction is reduced vagal activity, (4-6) which may also contribute to the phenotype of critically ill patients.(7) Concomitantly, impaired cardiometabolic responses to beta-adrenoceptor stimulation are robust negative prognostic biomarkers for survival in established critical illness. (8) However, unifying molecular mechanisms directly linking dysautonomia and cardiovascular dysfunction are lacking.

Neurohormonal dysregulation in disease states where biological variability is altered results in alterations in G-protein coupled receptor signalling, which regulate $\mathrm{G}$ protein-coupled receptor (GPCR) trafficking through phosphorylation-dependent and independent mechanisms.(9) Impaired GPCR signaling and trafficking is responsible for the loss of inotropy observed in experimental models of cardiac failure. GPCR-kinase 2 (GRK2) expression increases when cardiomyocytes are subjected to oxidative stress.(10) Neurohormonal activation associated with the loss of biologic variability may contribute to cardiac oxidative stress, since elevated plasma angiotensin generates reactive oxygen species (ROS) via activation of NADPH-oxidase (NOX-2) in cardiovascular pathology. (11) NOXderived, ROS-induced ROS release may disrupt mitochondrial electron transport chain or alter mitochondrial ionic homeostasis. (12)

In this study we tested the hypothesis that baroreflex dysfunction predisposes to the development of impaired cardiac contractility reported in established critical illness through altered expression of G-protein coupled receptor kinases and increased oxidative stress. To determine whether laboratory observations were clinically translational, we also identified impaired baroreflex sensitivity and associated parasympathetic dysfunction in patients undergoing major elective surgery to assess whether similar GPCR and autonomic dysfunction phenotypes were present in patients at risk of developing critical illness postoperatively.

\section{METHODS}

Detailed methods are described in the online supplement, including summaries demonstrating adherence to STROBE and ARRIVE guidelines. All research involving human participants was approved by Institutional Review Boards. Patients who underwent cardiopulmonary exercise testing as part of their enrollment in clinical trials at University College London Hospital (POM-O trial (13)) and Derriford Hospital, Plymouth (COMPETE-C trial (14); Supplementary Material) were assessed.

\section{Rodent Experiments}

All experiments were performed in accord with the UK Animals (Scientific Procedures) Act (1986) and ARRIVE guidelines (see Supplementary tables 2, 3 for summary of numbers and experiments). Male juvenile Sprague Dawley rats (Charles River, UK) and NOX-2 deficient and wild-type mice developed on a C57BL/6J background (King's College London British Heart Foundation Centre, London, United Kingdom) were used. In a randomized manner, carotid sinus and aortic depressor nerves were sectioned bilaterally (sino-aortic denervation: $\mathrm{SAD}$ ) in aseptic conditions under isoflurane anesthesia. (15) In sham-operated rats and mice, 
nerves were exposed and left intact. All experimental data were analyzed on an intention-totreat basis, and thus all nerve ablations were considered as achieving SAD.

\section{Transthoracic echocardiography}

Transthoracic echocardiography (Vevo 770, Visualsonics, Canada-mice; Vivid 7; GE

Healthcare, UK- rats) was performed under isoflurane anesthesia at 4-6 days and 3 weeks after SAD or sham surgery. Bolus doses of dobutamine were administered to assess cardiac contractility. All measurements were obtained by an investigator blinded to the identity of the experimental groups.

\section{Blood pressure measurements}

Tail cuff blood pressure measurements were made non-invasively (CODA, Kent Scientific, Torrington, VT, USA) under $2 \%$ isoflurane anaesthesia. All measurements were obtained by an investigator blinded to the identity of the experimental groups.

\section{Immunoblots}

Using antibodies against GRK2 (C-9) (Santa Cruz, sc-13143; (16)) GRK5 (C-20) (Santa Cruz, sc-565; (17)), pan-arrestin (Abcam, ab2914; (18)), $\beta$-Arrestin 1 (D8O3J; Cell Signalling Technology, \#12697), $\beta$-Arrestin 2 (C16D9; (Cell Signalling Technology,\#3857) and phospho-Troponin I (Cardiac) (Ser23/24) antibody (Cell Signalling Technology, \#4004), respective proteins were immunodetected from cell lysates prepared from cardiac ventricular tissue in rats and mice 2-3 weeks after the surgeries or mononuclear cells obtained from patients and prepared using Ficoll density gradient centrifugation. Proteins were resolved on SDS-PAGE gels. Densitometry determinations were calculated as the ratio between the protein and a-tubulin or phosphofructokinase protein expression.

\section{Quantitative PCR}

RNA was extracted from rodent left ventricles using the RNeasy Maxi Kit (Qiagen, Crawley, UK). Total RNA $(2 \mu \mathrm{g})$ was reverse-transcribed using a Taqman reverse transcriptase kit. cDNA (20 ng) was added to each well of a polymerase chain reaction array for quantitative polymerase chain reaction performed (Masterplex ${ }^{\circledR}$ realplex thermal cycler; Eppendorf, Germany). The comparative $\mathrm{Ct}$ method (2- $\Delta \Delta \mathrm{Ct}$ ) was used, with hypoxanthine-guanine phosphoribosyltransferase (HPRT) or cyclophilin as the housekeeping gene. For samples obtained from sham-operated and SAD animals, evaluation of (2- $\Delta \Delta \mathrm{Ct})$ was defined as the fold change in gene expression relative to the rat cerebral cortex. Primers are detailed in Supplementary Table 4.

\section{Patient studies}

\section{Assessment of Baroreflex sensitivity}

Using a validated sequence method (one beat lag) technique (19), spontaneous baroreflex sensitivity was measured in surgical patients who had previously undergone cardiopulmonary exercise testing from optimally damped intra-arterial pressure recordings (Lidco Plus, London, UK) obtained immediately preoperatively. No minimum thresholds 
were used for changes in systolic blood pressure or pulse interval; events were accepted provided the correlation coefficient between systolic blood pressure and pulse interval exceeded 0.85 . To avoid bias, all cardiopulmonary exercise and autonomic data analyses were undertaken by investigators blinded to clinical outcomes. Baroreflex values associated with increased mortality in multiple-organ dysfunction syndrome were stratified patients as having low/abnormal or normal BRS.(3)

\section{Assessment of parasympathetic vagal activity}

To determine parasympathetic (vagal) activity, two principle methods were employed. Firstly, heart rate recovery following exercise was measured as part of patients undergoing preoperative symptom-limited maximal cardiopulmonary exercise testing, with on-line breath-by-breath gas analysis monitoring. The anerobic threshold (AT), measured as body mass-corrected oxygen consumption $\left(\mathrm{ml} \cdot \mathrm{kg}^{-1} \cdot \mathrm{min}^{-1}\right)$ was used as a marker of aerobic fitness (20). AT was determined by the modified V slope method and confirmed with the ventilatory equivalents method. Abnormal heart rate recovery following exercise was defined according to BRS values and related to previous studies that demonstrated the negative prognostic power of a heart rate reduction of $\leq 2$ beats per minute from peak exercise to that measured one minute after cessation of exercise. (21) Secondly, Holter recordings were made preoperatively in a quiet environment using three-lead electrocardiographic recordings (Lifecard CF digital Holter monitors, Spacelabs Healthcare, Hertford, UK). Data cleaning and analysis was undertaken masked to cardiopulmonary exercise testingdata. Valid segments of recordings were identified from patients. Data quality criteria were in accordance with Task Force guidelines.(22) We assessed both time- and frequency domain measures from 5 min recordings in non-ventilated patients, although time-domain measures are preferable when only short-term recordings are possible since these are highly reproducible over time in a non-laboratory setting. (23) Two time-domain measures of parasympathetic activity were assessed: the square root of the mean of the sum of the squares of the successive differences between adjacent beat-to-beat intervals (root mean square of the successive differences, RMSSD); and the proportion of number of pairs of successive beat-to beat intervals that differ by more than $50 \mathrm{~ms}$, divided by total number of beat-to-beat intervals (pNN50). Spectral analysis of high frequency (HF) values, indicative of parasympathetic activity, was also performed (Spacelabs analysis software). To avoid bias, all autonomic data analyses were undertaken by investigators blinded to cardiopulmonary exercise test results and clinical outcomes.

\section{Intraoperative cardiac performance}

To assess the impact of baroreflex dysfunction on cardiac performance, we re-examined hemodynamic data from the COMPETE-C trial. (14) Comparison of intraoperative hemodynamic changes between groups measured by esophageal Doppler flow (CardioQ ${ }^{\mathrm{TM}}$, Deltex Medical, Chichester, UK) was restricted to those patients who underwent open laparotomy at timepoints free of acute surgical or anesthetic interventions.

\section{Patient outcomes}

Severity of postoperative complications in the COMPETE-C trial were categorized by two independent analyzers, according to the Clavien-Dindo grade (Supplementary Table 6). 
Diagnostic criteria for sepsis, critical care utilization and time to discharge have previously been reported by the COMPETE-C investigators.

Statistical methods-The primary outcome in the patient study was length of hospital stay stratified according to the presence or absence of heart rate recovery as defined by low BRS. Pre-defined secondary outcome measures were severe (>grade 3 Clavien-Dindo) complications, postoperative sepsis and requirement at any point for admission to critical care. Power calculations for the clinical study design are provided in online supplement. Experimental rodent studies were designed in accordance with ARRIVE. For continuous data, normality of distribution was assessed and, where appropriate, analysed with ANOVA. Nonparametric data were analysed with the Mann-Whitney U-test. Length of hospital stay was estimated using the Kaplan-Meier method and analysed using the Cox proportional hazard model (taking into account autonomic phenotype and randomization arm in the COMPETE-C trial). All reported $\mathrm{p}$ values are two-sided. Statistical analyses were performed using NCSS 8 (Kaysville, UT). Median values (interquartile range) are presented, unless stated otherwise. Significance was accepted at p values $₫ 0.05$.

\section{RESULTS}

\section{Baroreflex dysfunction results in impaired cardiac contractility}

We first determined whether baroreflex dysfunction (3 weeks after SAD surgery) impairs cardiac function. We chose the 3 week timepoint to reflect the typical period over which chronic critical illness becomes established. Baroreflex dysfunction was induced by bilateral sectioning of aortic and carotid sinus nerves (hereafter, termed SAD: sino-aortic denervation). Rats which underwent SAD surgery appeared clinically unwell as adjudged by their appearance (mild piloerection, hunched) in the first 3 days following nerve ablation. During the subsequent 3 weeks, SAD rats gained less weight (Figure 1A). Using a protocol (Figure 1B) similar to that used in humans (24), we found that established SAD assessed 3 weeks after the surgery was associated with an impaired inotropic response (Figure 1C). The increase in cardiac output as a result of preserved contractile response to dobutamine was only observed in sham-operated rats. Dobutamine elicited a relative tachycardia in SAD rats yet failed to elicit an inotropic response (Supplementary Figure 1).

\section{Enhanced cardiac GRK expression following baroreflex dysfunction}

In view of the loss of the beta- 1 adrenoceptor response to dobutamine in both human critical illness (8) and our baroreflex dysfunction model, we hypothesized that cardiac impairment is due to impaired GPCR signaling similar to that observed in experimental models of cardiac failure. (25) Protein expression of both GRK-2 (Figure 2A) and $\beta$-arrestin-1 (Figure 2B) was upregulated in SAD rat ventricular cardiomyocytes, whereas no change was seen in GRK-5 expression (Figure 2C, D). SAD is known to be associated with transient (but not chronic) elevations in the level of circulating angiotensin. (26) We therefore hypothesised that angiotensin is likely to be responsible for producing cardiac dysfunction in SAD model through angiotensin-induced reactive oxygen species (ROS) generation, via activation of NOX-2.(11) We found that expression of angiotensin-II receptor 1a is upregulated in conditions of baroreflex dysfunction (Figure 2E). 


\section{NOX2 activity triggers GRK2 expression in conditions of baroreflex dysfunction}

We next tested the hypothesis that SAD impairs cardiac function through angiotensin induced oxidative stress, via activation of NADPH-oxidase (NOX-2). Transthoracic echocardiography showed that NOX2 deficiency prevents cardiac impairment that developed in wild-type SAD mice (Figure $3 \mathrm{~A}$ ), where a significant $20 \%$ reduction in ejection fraction was observed (Figure 3B) despite similar blood pressures between genotypes (Figure 3C). In the failing heart, PKA activity is altered as a result of dysfunctional beta-adrenoreceptor signalling.(9) This results in decreased troponin I phosphorylation (ser23/24), which was lower in wild-type mice following SAD (Figure 3D). Quantitative PCR of cardiomyocytes showed that both GRK-2 (Figure 3E) and GRK-5 (Figure 3F) mRNA was only upregulated in wild-type mice ten days after SAD, but not in NOX2-deficient murine cardiomyocytes. Protein expression of GRK-2, but not GRK-5, mirrored the transcriptomic changes observed in wild-type murine cardiomyocytes following SAD (Figure 3G). We also observed differences in beta-arrestin 1 mRNA transcription in NOX2-deficient murine cardiomyocytes following SAD (Figure 3I-J)

\section{Baroreflex dysfunction is associated with impaired vagal activity in patients at risk of developing postoperative critical illness}

Impaired baroreflex sensitivity is associated with generalized autonomic dysfunction, including reduced parasympathetic (vagal) activity in both laboratory models (6) and patients. (7) We confirmed that baroreflex dysfunction is associated with impaired vagal activity in this patient population (Figure 4A-E) by assessing five measures of parasympathetic activity in patients with normal and low ( $56 \mathrm{~ms} . \mathrm{mmHg}^{-1}$ ) spontaneous baroreflex sensitivity values. First, we assessed exercise-induced heart rate recovery in a cohort of matched patients with similar aerobic exercise capacity (Table 1). Patients with low BRS had heart rate recovery values (Figure 5A) similar to those defined by epidemiologic (21) and physiologic (27) studies that have identified heart rate recovery $\checkmark 2 \mathrm{bpm}$ as a robust independent prognosticator for worse clinical outcomes. No pre-defined medical (cardiovascular) co-morbidities were associated with heart rate recovery $₫ 2 \mathrm{bpm}$, including diabetes mellitus (Table 1). Three different time-domain measures of parasympathetic activity obtained from Holter recordings made preoperatively were similarly consistent, with impaired BRS associated with lower values for RR-interval (Figure 4B), RMSSD (Figure 4C) and pNN50 (Figure 4D). Spectral analysis of the high frequency component of heart rate variability also showed lower values for patients with BRS $5 \mathrm{~ms} . \mathrm{mmHg}^{-1}$ (Figure 4E). In a second separate patient cohort (Plymouth), the thresholds defined by BRS from the derivation cohort gave similar HRR values (Figure 4F). Thus, every parasympathetic measure undertaken demonstrated that low vagal activity was strongly associated with impaired baroreflex sensitivity.

\section{Baroreflex dysfunction in patients is associated with elevated expression of GRK2 in circulating mononuclear leukocytes}

As baroreflex dysfunction recapitulates many features of cellular dysfunction synonymous with established critical illness, we next tested whether we could find evidence for this in circulating leukocytes which are exposed to a similar neurohormonal environment as 
cardiomyocytes- as has been shown in cardiac failure patients.(9) GRK2 expression in circulating mononuclear cells has been shown to parallel expression of this GPCR recycling protein in cardiac (left ventricular) tissue biopsies in patients with both heart failure (28) and systemic arterial hypertension.(29) We found that parasympathetic dysfunction was associated with increased GRK2 expression in circulating mononuclear cells (Figure 4G) obtained preoperatively from surgical patients (Figure $4 \mathrm{H}$ ).

\section{Autonomic dysfunction is associated with failure to increase cardiac contractility intraoperatively}

We next asked whether autonomic dysfunction may explain the failure to achieve optimal hemodynamic performance following major surgery, which is associated with increased morbidity and prolonged hospital stay.(8) The COMPETE-C trial (14) failed to find any benefit of stroke volume optimization intraoperatively, and we therefore considered whether a failure to increase cardiac contractility in patients with autonomic dysfunction may account for this. We therefore evaluated the relationship between autonomic dysfunction and intraoperative hemodynamic function by re-analyzing data from the COMPETE-C trial, where intraoperative hemodynamic data was collected in patients who had undergone preoperative cardiopulmonary exercise testing. Using the same threshold values we obtained for heart rate recovery as a surrogate for baroreflex dysfunction (Figure 4F), low HRR ( $\triangle 10 \mathrm{bpm}$ ) was observed in $81 / 249$ (32.5\%) patients who underwent preoperative cardiopulmonary exercise testing as part of the screening process for the COMPETE-C trial. Having established the prevalence of autonomic dysfunction as defined by abnormal heart rate recovery in this cohort (blinded to perioperative details), we next assessed the intraoperative hemodynamic performance of those patients who subsequently completed the COMPETE-C randomized controlled trial protocol (Table 2; $\mathrm{n}=175$ ). Despite similar perioperative heart rates (Figure 5A) and mean arterial pressure (Figure 5B), cardiac contractility, measured using the esophageal Doppler, did not alter in patients with autonomic dysfunction (Figure 5C). The failure of patients with parasympathetic autonomic dysfunction to achieve optimal hemodynamic performance was associated with an excess of serious (Clavien-Dindo grade 3 ) postoperative complications (relative risk: 2.60 (95\% CI: 1.18-5.74); $\mathrm{p}=0.014$ ), more frequent episodes of sepsis (relative risk:2.70 [1.49-4.93]; $\mathrm{p}=0.001$ ), increased use of critical care resources (relative risk:2.22 [1.15-4.30]; $\mathrm{p}=0.016$ ) and prolonged hospital stay (unadjusted relative risk:1.42 (95\%CI: 1.05-1.92); $\mathrm{p}=0.02$; Figure 5D). Cox regression analysis showed that the association between prolonged hospital stay and parasympathetic autonomic dysfunction remained when controlling for hemodynamic therapy (relative risk:1.59 (95\% CI: 1.13-2.24); $\mathrm{p}=0.006$ ). Parasympathetic autonomic dysfunction was also associated with higher mortality, extending to 600 days postoperatively (hazard ratio: 1.16 [0.84-1.60]; $\mathrm{p}=0.007$; Figure 5E).

\section{DISCUSSION}

Autonomic dysfunction is associated with every stage of critical illness although its role as a trigger of this maladaptive state, rather than merely being a marker, has remained largely unexplored. By taking an integrative experimental approach, we found that by solely disrupting afferent autonomic signalling results in profound clinically-relevant, cellular 
features synonymous with the cardiac phenotype of critical illness. Patients with pre-existing autonomic dysfunction (as measured by assessment of baroreflex and parasympathetic activity) undergoing elective major surgery manifest a similar phenotype, characterized by molecular and physiologic signatures of impaired cardiac contractility. This dysautonomic phenotype is linked to an excess of infectious complications. This autonomic reinterrogation of the COMPETE-C trial explains an apparent disconnect between limited intraoperative cardiac performance and postoperative outcome.

Cardiac dysfunction is a key feature of critical illness that correlates with poor outcomes. (30) An inability to mount an appropriate cardio-metabolic response to beta-adrenoceptor stimulation (using a dobutamine challenge test) is associated with increased mortality in critical illness $(8,24)$. Interestingly, autonomic dysfunction recapitulates this maladaptive phenotype, both in surgical patients with established autonomic dysfunction intraoperatively and in animal model with experimentally-induced autonomic dysfunction. Established cardiac failure is characterized at the molecular level by significant up-regulation of GRK2 (31), a member of the GRK family of serine/threonine protein kinases that phosphorylate and desensitize GPCRs, which mediate the effects of beta-adrenoceptor stimulation. (9) In contrast, acute (32) and chronic inflammation associated with rheumatoid arthritis (33) have been linked with downregulation of GRK2 levels in monocytes. Following agonist activation, GRKs rapidly phosphorylate GPCRs; through binding of arrestins to the phosphorylated receptors, the receptor is uncoupled from the G protein. (34) Prolonged agonist stimulation leads to persistent homologous receptor desensitization and hence the loss of receptor responsiveness. GRK2 inhibits pro-contractile signalling pathways by phosphorylating the beta-adrenoceptors and promoting beta-arrestin binding. (9) Mice lacking $\beta$-arrestin lose cardioprotective mechanisms that counteract prolonged mechanical stretch (35). Thus, the impaired baseline function and inotropic responses to betaadrenoceptor stimulation in the rat SAD model of baroreflex dysfunction are consistent with increased expression of GRK2 in cardiomyocytes. The upregulation of $\beta$-arrestin expression most likely reflects compensatory cell survival signaling strategy in the rat SAD model, triggered through activation of common GPCR pathways as a result of baroreflex dysfunction -induced activation of the angiotensin system. Consistent with this hypothesis, we also found no compensatory increase in $\beta$-arrestin in the murine SAD model, where a lower ejection fraction was observed in the wild-type mice with cardiac contractility was found to be markedly worse than in the rat model. However, in the murine SAD model we also found a compensatory upregulation of GRK5(36) in wild-type mice; increased levels of GRK-5 correlate positively with lower morbidity/mortality in human heart failure.(37)

Resting plasma levels of angiotensin and vasopressin in sino-aortic denervated rats were previously found to be similar to that of sham-operated animals; however, various stressors elicit marked increases in the plasma levels of both hormones. (38) We found that transcription of angiotensin-II receptor type 1 is upregulated in SAD rats, consistent with the observation that expression of this receptor is positively correlated with blood pressure throughout multiple neuroendocrine tissues in the spontaneously hypertensive rat. (39) Angiotensin infused at levels that do not alter systemic arterial blood pressure induce NOX2-mediated cardiac hypertrophy within two weeks (40) NOX-2 activation also contributes to myocardial hypertrophy induced by other humoral factors commonly 
upregulated in critical illness.(11) Impaired contractility via excessive activation of NOX2 may be due to increased expression of L-type calcium channels (41) and/or pathological changes in mechanosensitive stretch-induced calcium release.(42) Pathologically excessive activation of NOX-2 by angiotensin actions in the sarcolemma and T-tubules impairs ROSinduced sensitization of the ryanodine receptor which regulates release of calcium from the sarcoplasmic reticulum to enhance calcium-induced calcium release. (42) Thus, pathologic activation of NOX-2 may prevent optimal calcium release in response to stretch, a critical mechanism which controls cardiac function as described by the Frank-Starling law of the heart. In the context of critical illness, deficiency of gp91phox attenuates myocardial depression following endotoxemia, (43) which also triggers rapid and profound alterations in baroreflex control.(44) In this study we employed both rat and mouse SAD models. Sinoaortic denervation in mice increases blood pressure variability dramatically (45) although some studies have reported, in contrast to other species, an increase in resting arterial blood pressure early after SAD.(5) This may affect cardiac contractility independently of other direct (neurohormonal) alterations in cardiac physiology. Thus, timing appears to be an important factor in the expression of a variable cardiovascular phenotype reported following SAD in mice, and we, therefore, chose the 2-3 week timepoint as this appears to be compatible with the development of established critical illness. Other protective mechanisms through which the autonomic nervous system provides the neural conduit to enable interorgan crosstalk cannot be excluded, including the prevention of gut barrier failure (46). Nevertheless, both mouse and rat SAD models demonstrated similar pattern of myocardial GRK2 upregulation, suggesting that this cellular pathophysiological process occurs independently of blood pressure changes. Consistent with this assertion, our results demonstrate that patients with parasympathetic autonomic dysfunction also exhibited elevated GRK2 expression in circulating mononuclear cells, independent of blood pressure.

This study has a number of clinical implications. The autonomic phenotype we describe provides a rapidly testable, integrative, patient-centered physiological paradigm to enhance the clinical stratification of risk, elucidate mechanisms of established therapeutics and devise novel interventional targets. The BRS values reported herein are remarkably similar to those previously found to predict increased risk of mortality following multiple-organ dysfunction syndrome.(3) We provide mechanistic translational data that provides a biologic rationale that helps explain why inferior cardiac performance during stressor situations may lead to further clinical complications. Interestingly, several pharmacologic agents have reversed/ attenuated this pathological autonomic phenotype in other clinical areas, for example heart failure- another syndrome similarly characterized by persistent neurohormonal activation and a pro-inflammatory state.(47) Our data also provide mechanistic insights into the results of recent exploratory trials which demonstrated better outcomes in critical illness, even though underlying mechanisms remained poorly understood. For example, beta-1 adrenoceptor blockade (48) and alpha-2 agonism (49) exert significant autonomic effects that, in part, can reverse/prevent several features of the cardiac phenotype that develops during critical illness. Avoiding pharmacological and/or therapeutic interventions that promote dysautonomia and consequently increase iatrogenic complications is likely to be beneficial, including the avoidance of prolonged bed rest by adopting early mobilization of critically ill patients (50). 
Laboratory and clinical epidemiologic data demonstrated that augmented vagal (parasympathetic) activity limits cardiovascular morbidity and mortality. (51) Importantly, the presence of parasympathetic autonomic dysfunction in the human surgical study cohort was not associated with pathologies traditionally linked to autonomic dysfunction such as diabetes mellitus. This is perhaps unsurprising given the link between autonomic parasympathetic dysfunction and all-cause mortality. Epidemiologic data demonstrating an association between hypertension and hospitalization for sepsis (52) is also consistent with the autonomic phenotype described here.

In summary, we suggest that baroreflex autonomic dysfunction mimics key cardiovascular aspects of critical illness. These findings offer the potential for novel therapeutic paradigms that could limit the excess morbidity and mortality associated with critical illness. Similarly, avoiding or reducing iatrogenic, off-target consequences of treatments that provoke or sustain autonomic dysfunction may also be beneficial.

\section{Acknowledgments}

Ben Rimmer for data collection at Derriford Hospital; Mary Elphinstone, Paul Erasmus, Daryl Thorp-Jones, Gavin Werrett for performing cardiopulmonary exercise testingtesting at Derriford Hospital; Amber Emmerson (Kings College) for murine husbandry; Professor K. Michael Spyer for helpful discussions, Sadaf Iqbal, Laura Gallego Paredes and Anna Reyes for research nurse support, and all the surgical teams and patients for participating in these studies.

Support: Academy Medical Sciences/Health Foundation Clinician Scientist Award; National Institute of Academic Anaesthesia/British Journal of Anaesthesia Basic Science career development award (GLA); British Heart Foundation Program Grant (RG/14/4/30736; AVG, GLA). AVG is a Wellcome Trust Senior Research Fellow. GLA and AVG are supported by British Heart Foundation Programme Grant. GM held an BJA/RCOA Project grant. MS is a NIHR Senior Investigator. AMS is Director, British Heart Foundation Centre for Excellence, Kings College, London, UK. Part of this work was undertaken at UCLH/UCL who received a proportion of funding from the UK Department of Health's NIHR Biomedical Research Centres funding scheme.

\section{Supplementary Material}

Refer to Web version on PubMed Central for supplementary material.

\section{References}

1. Godin PJ, Buchman TG. Uncoupling of biological oscillators: a complementary hypothesis concerning the pathogenesis of multiple organ dysfunction syndrome. Crit Care Med. 1996; 24(7): 1107-1116. [PubMed: 8674321]

2. Convertino VA, Doerr DF, Eckberg DL, et al. Head-down bed rest impairs vagal baroreflex responses and provokes orthostatic hypotension. J Appl Physiol. 1990; 68(4):1458-1464. [PubMed: 2347788]

3. Schmidt H, Muller-Werdan U, Hoffmann T, et al. Autonomic dysfunction predicts mortality in patients with multiple organ dysfunction syndrome of different age groups. Crit Care Med. 2005; 33(9):1994-2002. [PubMed: 16148471]

4. Silva LE, Rodrigues FL, de Oliveira M, et al. Heart rate complexity in sinoaortic-denervated mice. Exp Physiol. 2014

5. Rodrigues FL, de Oliveira M, Salgado HC, et al. Effect of baroreceptor denervation on the autonomic control of arterial pressure in conscious mice. Exp Physiol. 2011; 96(9):853-862. [PubMed: 21666038]

6. Soares PP, Porto CS, Abdalla FM, et al. Effects of rat sinoaortic denervation on the vagal responsiveness and expression of muscarinic acetylcholine receptors. Journal of cardiovascular pharmacology. 2006; 47(3):331-336. [PubMed: 16633073] 
7. Thayer JF, Lane RD. The role of vagal function in the risk for cardiovascular disease and mortality. Biol Psychol. 2007; 74(2):224-242. [PubMed: 17182165]

8. Collin S, Sennoun N, Levy B. Cardiovascular and metabolic responses to catecholamine and sepsis prognosis: a ubiquitous phenomenon? Critical Care (London, England). 2008; 12(2):118.

9. Lymperopoulos A, Rengo G, Koch WJ. Adrenergic nervous system in heart failure: pathophysiology and therapy. Circ Res. 2013; 113(6):739-753. [PubMed: 23989716]

10. Chen M, Sato PY, Chuprun JK, et al. Prodeath signaling of G protein-coupled receptor kinase 2 in cardiac myocytes after ischemic stress occurs via extracellular signal-regulated kinase-dependent heat shock protein 90-mediated mitochondrial targeting. Circ Res. 2013; 112(8):1121-1134. [PubMed: 23467820]

11. Zhang M, Perino A, Ghigo A, et al. NADPH oxidases in heart failure: poachers or gamekeepers? Antioxid Redox Signal. 2013; 18(9):1024-1041. [PubMed: 22747566]

12. Zinkevich NS, Gutterman DD. ROS-induced ROS release in vascular biology: redox-redox signaling. Am J Physiol Heart Circ Physiol. 2011; 301(3):H647-653. [PubMed: 21685266]

13. Ackland GL, Iqbal S, Paredes LG, et al. Individualised oxygen delivery targeted haemodynamic therapy in high-risk surgical patients: a multicentre, randomised, double-blind, controlled, mechanistic trial. Lancet Respir Med. 2015; 3(1):33-41. [PubMed: 25523407]

14. Challand C, Struthers R, Sneyd JR, et al. Randomized controlled trial of intraoperative goaldirected fluid therapy in aerobically fit and unfit patients having major colorectal surgery. $\mathrm{Br} \mathbf{J}$ Anaesth. 2012; 108(1):53-62. [PubMed: 21873370]

15. Krieger EM. Neurogenic Hypertension in the Rat. Circ Res. 1964; 15:511-521. [PubMed: 14243890]

16. Liu X, Ma B, Malik AB, et al. Bidirectional regulation of neutrophil migration by mitogenactivated protein kinases. Nat Immunol. 2012; 13(5):457-464. [PubMed: 22447027]

17. Petrich A, Mann A, Kliewer A, et al. Phosphorylation of threonine 333 regulates trafficking of the human sst5 somatostatin receptor. Mol Endocrinol. 2013; 27(4):671-682. [PubMed: 23418396]

18. Law IK, Murphy JE, Bakirtzi K, et al. Neurotensin-induced proinflammatory signaling in human colonocytes is regulated by beta-arrestins and endothelin-converting enzyme-1-dependent endocytosis and resensitization of neurotensin receptor 1. J Biol Chem. 2012; 287(18):1506615075. [PubMed: 22416137]

19. Laude D, Elghozi JL, Girard A, et al. Comparison of various techniques used to estimate spontaneous baroreflex sensitivity (the EuroBaVar study). Am J Physiol Regul Integr Comp Physiol. 2004; 286(1):R226-231. [PubMed: 14500269]

20. ATS/ACCP Statement on cardiopulmonary exercise testing. Am J Respir Crit Care Med. 2003; 167(2):211-277. [PubMed: 12524257]

21. Cole CR, Blackstone EH, Pashkow FJ, et al. Heart-rate recovery immediately after exercise as a predictor of mortality. The New England journal of medicine. 1999; 341(18):1351-1357. [PubMed: 10536127]

22. Novak V, Saul JP, Eckberg DL. Task Force report on heart rate variability. Circulation. 1997; 96(3): 1056-1057. [PubMed: 9264529]

23. Sinnreich R, Kark JD, Friedlander Y, et al. Five minute recordings of heart rate variability for population studies: repeatability and age-sex characteristics. Heart. 1998; 80(2):156-162. [PubMed: 9813562]

24. Rhodes A, Lamb FJ, Malagon I, et al. A prospective study of the use of a dobutamine stress test to identify outcome in patients with sepsis, severe sepsis, or septic shock. Crit Care Med. 1999; 27(11):2361-2366. [PubMed: 10579249]

25. Volkers M, Weidenhammer C, Herzog N, et al. The inotropic peptide betaARKct improves betaAR responsiveness in normal and failing cardiomyocytes through G(betagamma)-mediated L-type calcium current disinhibition. Circ Res. 2011; 108(1):27-39. [PubMed: 21106943]

26. Shan ZZ, Dai SM, Fang F, et al. Changes of central norepinephrine, beta-endorphin, LEUenkephalin, peripheral arginine-vasopressin, and angiotensin II levels in acute and chronic phases of sino-aortic denervation in rats. J Cardiovasc Pharmacol. 2004; 43(2):234-241. [PubMed: 14716211] 
27. Imai K, Sato H, Hori M, et al. Vagally mediated heart rate recovery after exercise is accelerated in athletes but blunted in patients with chronic heart failure. Journal of the American College of Cardiology. 1994; 24(6):1529-1535. [PubMed: 7930286]

28. Iaccarino G, Barbato E, Cipolletta E, et al. Elevated myocardial and lymphocyte GRK2 expression and activity in human heart failure. Eur Heart J. 2005; 26(17):1752-1758. [PubMed: 16055494]

29. Gros R, Benovic JL, Tan CM, et al. G-protein-coupled receptor kinase activity is increased in hypertension. J Clin Invest. 1997; 99(9):2087-2093. [PubMed: 9151780]

30. Parker MM, Shelhamer JH, Bacharach SL, et al. Profound but reversible myocardial depression in patients with septic shock. Ann Intern Med. 1984; 100(4):483-490. [PubMed: 6703540]

31. Ungerer M, Parruti G, Bohm M, et al. Expression of beta-arrestins and beta-adrenergic receptor kinases in the failing human heart. CircRes. 1994; 74(2):206-213.

32. Hagen SA, Kondyra AL, Grocott HP, et al. Cardiopulmonary bypass decreases G protein-coupled receptor kinase activity and expression in human peripheral blood mononuclear cells. Anesthesiology. 2003; 98(2):343-348. [PubMed: 12552191]

33. Lombardi MS, Kavelaars A, Schedlowski M, et al. Decreased expression and activity of G-proteincoupled receptor kinases in peripheral blood mononuclear cells of patients with rheumatoid arthritis. FASEB J. 1999; 13(6):715-725. [PubMed: 10094932]

34. Pitcher JA, Freedman NJ, Lefkowitz RJ. G protein-coupled receptor kinases. Annu Rev Biochem. 1998; 67:653-692. [PubMed: 9759500]

35. Patel PA, Tilley DG, Rockman HA. Physiologic and cardiac roles of beta-arrestins. JMolCell Cardiol. 2009; 46(3):300-308.

36. Salazar NC, Vallejos X, Siryk A, et al. GRK2 blockade with betaARKct is essential for cardiac beta2-adrenergic receptor signaling towards increased contractility. Cell Commun Signal. 2013; 11:64. [PubMed: 23984976]

37. Liggett SB, Cresci S, Kelly RJ, et al. A GRK5 polymorphism that inhibits beta-adrenergic receptor signaling is protective in heart failure. Nat Med. 2008; 14(5):510-517. [PubMed: 18425130]

38. Miao CY, Tao X, Gong K, et al. Arterial remodeling in chronic sinoaortic-denervated rats. Journal of cardiovascular pharmacology. 2001; 37(1):6-15. [PubMed: 11152375]

39. Reja V, Goodchild AK, Phillips JK, et al. Upregulation of angiotensin AT1 receptor and intracellular kinase gene expression in hypertensive rats. Clin Exp Pharmacol Physiol. 2006; 33(8): 690-695. [PubMed: 16895541]

40. Bendall JK, Cave AC, Heymes C, et al. Pivotal role of a gp91(phox)-containing NADPH oxidase in angiotensin II-induced cardiac hypertrophy in mice. Circulation. 2002; 105(3):293-296. [PubMed: 11804982]

41. Zeng Q, Zhou Q, Yao F, et al. Endothelin-1 regulates cardiac L-type calcium channels via NAD(P)H oxidase-derived superoxide. J Pharmacol Exp Ther. 2008; 326(3):732-738. [PubMed: 18539650]

42. Prosser BL, Ward CW, Lederer WJ. X-ROS signaling: rapid mechano-chemo transduction in heart. Science. 2011; 333(6048):1440-1445. [PubMed: 21903813]

43. Li X, Li Y, Shan L, et al. Over-expression of calpastatin inhibits calpain activation and attenuates myocardial dysfunction during endotoxaemia. Cardiovasc Res. 2009; 83(1):72-79. [PubMed: 19318376]

44. Koyama S, Santiesteban HL, Ammons WS, et al. Centrally mediated hypotensive effect of E coli endotoxin in the anesthetized cat. Circ Shock. 1982; 9(6):557-570. [PubMed: 6758978]

45. Martinka P, Fielitz J, Patzak A, et al. Mechanisms of blood pressure variability-induced cardiac hypertrophy and dysfunction in mice with impaired baroreflex. Am J Physiol Regul Integr Comp Physiol. 2005; 288(3):R767-776. [PubMed: 15563577]

46. Costantini TW, Krzyzaniak M, Cheadle GA, et al. Targeting alpha-7 nicotinic acetylcholine receptor in the enteric nervous system: a cholinergic agonist prevents gut barrier failure after severe burn injury. Am J Pathol. 2012; 181(2):478-486. [PubMed: 22688057]

47. Zhang Y, Popovic ZB, Bibevski S, et al. Chronic vagus nerve stimulation improves autonomic control and attenuates systemic inflammation and heart failure progression in a canine high-rate pacing model. Circ Heart Fail. 2009; 2(6):692-699. [PubMed: 19919995] 
48. Ackland GL, Yao ST, Rudiger A, et al. Cardioprotection, attenuated systemic inflammation, and survival benefit of beta1-adrenoceptor blockade in severe sepsis in rats. Crit Care Med. 2010; 38(2):388-394. [PubMed: 19829100]

49. Pandharipande PP, Pun BT, Herr DL, et al. Effect of sedation with dexmedetomidine vs lorazepam on acute brain dysfunction in mechanically ventilated patients: the MENDS randomized controlled trial. JAMA: The Journal of the American Medical Association. 2007; 298(22):2644-2653. [PubMed: 18073360]

50. Schweickert WD, Pohlman MC, Pohlman AS, et al. Early physical and occupational therapy in mechanically ventilated, critically ill patients: a randomised controlled trial. Lancet. 2009; 373(9678):1874-1882. [PubMed: 19446324]

51. De Ferrari GM, Schwartz PJ. Vagus nerve stimulation: from pre-clinical to clinical application: challenges and future directions. Heart failure reviews. 2011; 16(2):195-203. [PubMed: 21165697]

52. Wang HE, Shapiro NI, Griffin R, et al. Chronic medical conditions and risk of sepsis. PLoS One. 2012; 7(10):e48307. [PubMed: 23118977] 


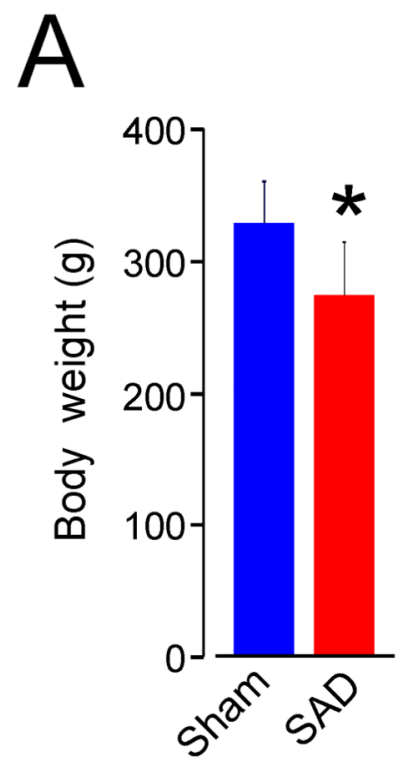

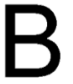

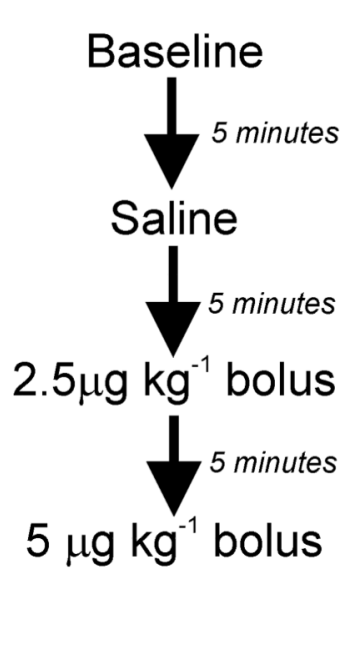

C

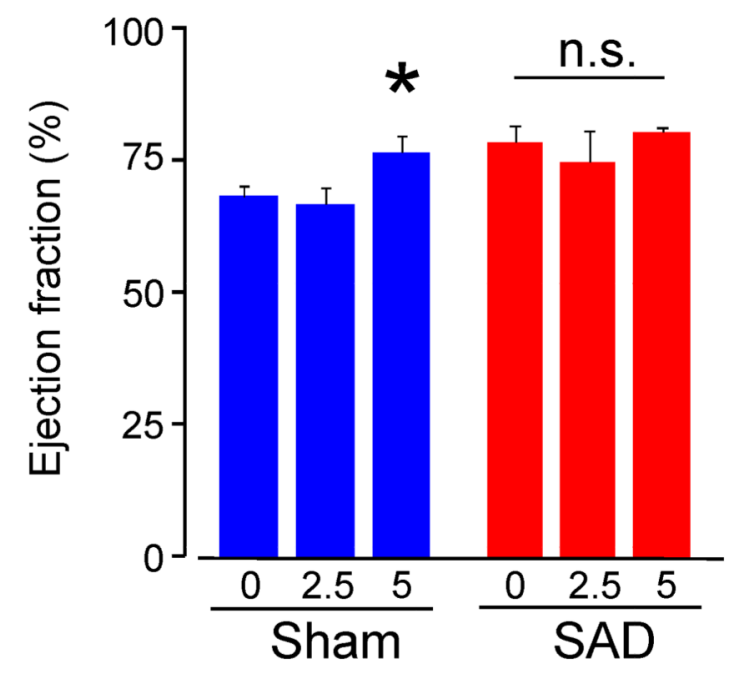

Figure 1.

Cardiac performance in experimental afferent autonomic dysfunction in rats. (A) Loss of weight following sino-aortic denervation (SAD). (B) Summary of dobutamine protocol to assess cardiac performance at three weeks following SAD. (C) Dobutamine-induced increases in left ventricular ejection fraction are observed in sham operated rats 3 weeks after nerve ablation surgery. Data are presented as mean $\pm S E(n=4-6$ rats per group). Asterisk indicates $\mathrm{p}<0.05$; n.s. - not significant. 


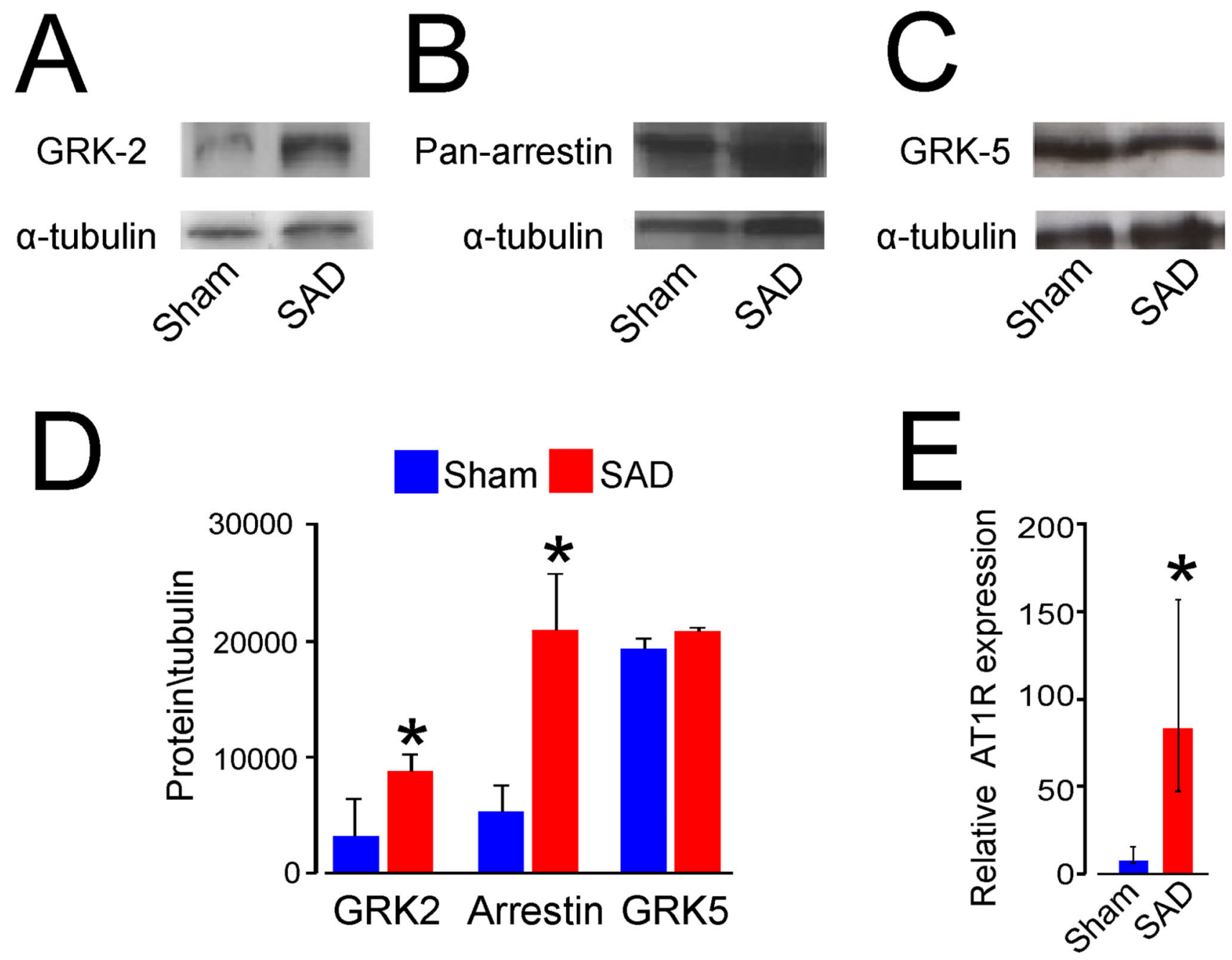

Figure 2.

GPCR signalling protein expression in left ventricular tissue following 3 weeks of experimental afferent autonomic dysfunction. (A) Representative immunoblot for rat cardiomyocyte GRK-2 (B) cardiomyocyte $\beta$-arrestin-1. (C) cardiomyocyte GRK-5 protein expression. (D) Group densitometry data for GRK2, $\beta$-arrestin-1 and GRK5 in sham and $\mathrm{SAD}$ rat left ventricular tissue, referenced to a-tubulin (mean $\pm \mathrm{SE}$ ). (E) Quantitative PCR showing upregulation of ATII-1R mRNA expression in SAD rat ventricular cardiomyocytes (referenced to LN229 neuronal cell line expression). *Significant difference $(\mathrm{P}<0.05)$; $\mathrm{n}=4-6 /$ group. 

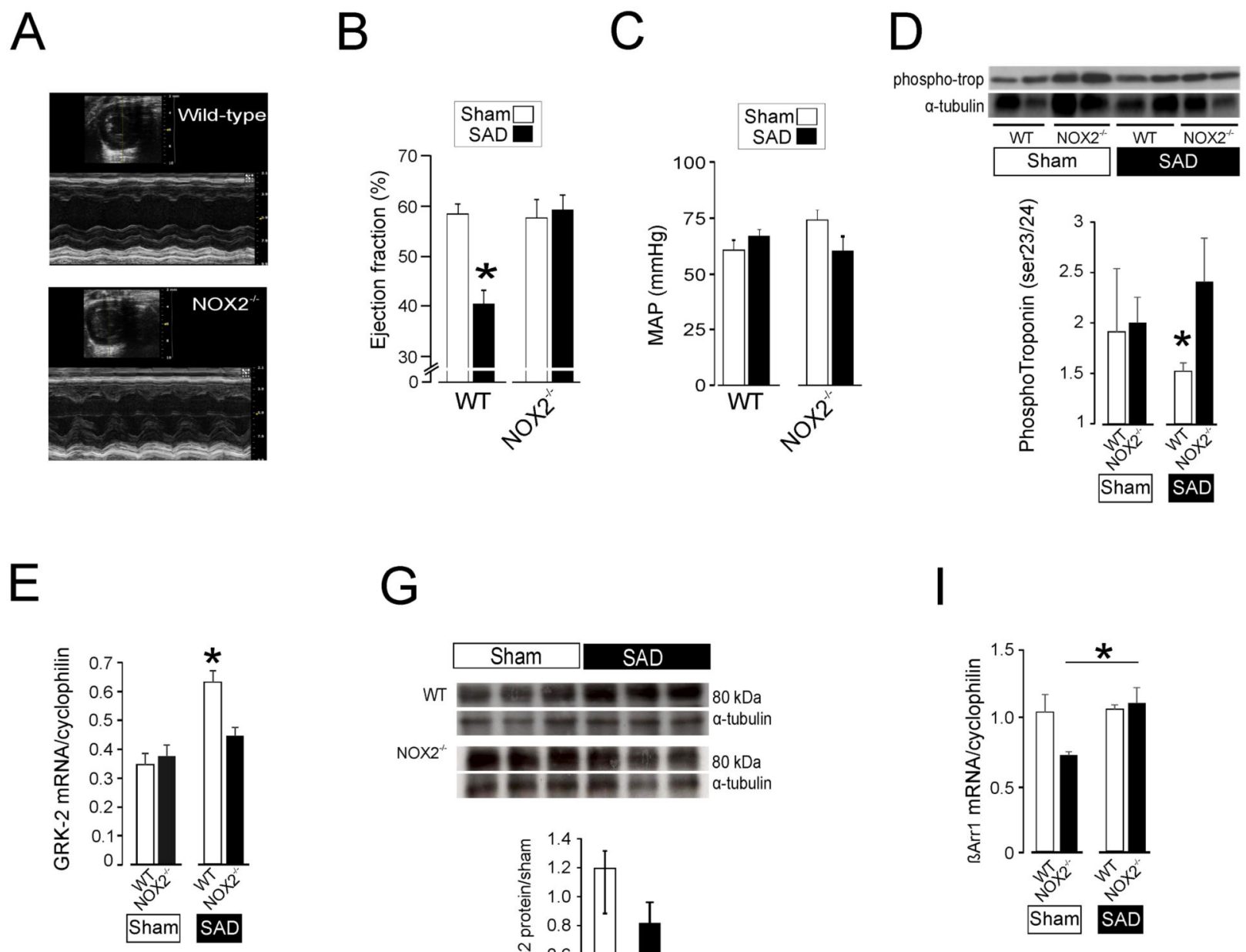

\section{G}
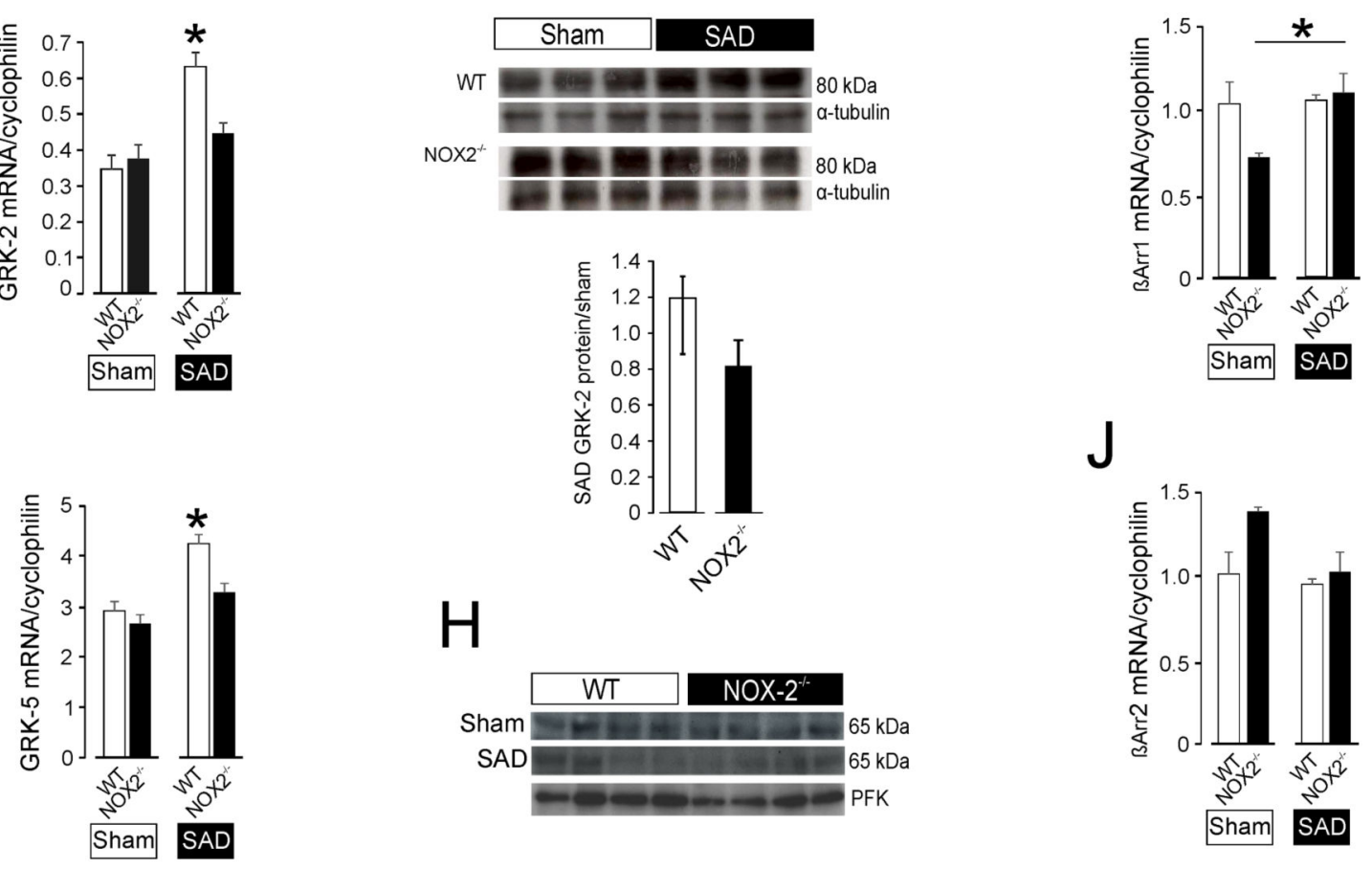

Figure 3.

Afferent autonomic dysfunction promotes GRK2 over-expression via activation of NOX-2. (A) Representative M-mode echocardiography image showing impaired baseline contractility 26 days after sino-aortic denervated in a wild-type mouse, but preserved cardiac contractility 26 days after sino-aortic denervation in a representative NOX2 mutant mouse. (B) Population data for ejection fraction in wild-type or NOX-2-/- mouse following either sham or sino-aortic denervation procedure. 3-5/experimental group; mean \pm SE. *Significant difference $(\mathrm{P}<0.05)$. (C) Similar mean arterial pressure (measured by tail-cuff occlusion) in 
anesthetized wild-type or NOX-2-/- mice. (D) Immunoblot showing reduction in phosphotroponin (Ser23/24) in wild-type cardiomyocytes 10 days after bilateral sino-aortic denervation. (E) Quantitative PCR demonstrating upregulation of GRK2 mRNA expression in SAD wild-type cardiomyocytes (referenced to expression in mouse brain cortex samples). *Significant difference ( $\mathrm{P}<0.01)$; $\mathrm{n}=4$ /group. (F) Quantitative PCR demonstrating upregulation of GRK5 mRNA expression in SAD wild-type cardiomyocytes, same samples as shown in panel $\mathrm{E}$ (referenced to expression in mouse brain cortex samples). *Significant difference ( $\mathrm{P}<0.01)$; $\mathrm{n}=4$ /group. (G) Representative immunoblot for GRK2 in murine wildtype or NOX-2/- ventricular cardiomyocytes, ten days after sham surgery or sino-aortic denervation (SAD). Population data show change in GRK2 protein levels for SAD wild-type or NOX $-2^{-/-}$ventricular cardiomyocytes, as relative change over mean sham protein levels (* $\mathrm{P}=0.07$, unpaired t-test, $\mathrm{n}=4$-6/group). (H) Representative immunoblot for GRK5 in murine wild-type or NOX-2-/- ventricular cardiomyocytes, ten days after sham surgery or sino-aortic denervation (SAD). (I) Quantitative PCR for $\beta$-arrestin 1 mRNA expression in SAD wildtype cardiomyocytes (referenced to expression in mouse brain cortex samples). *Significant difference ( $\mathrm{P}<0.05)$; $\mathrm{n}=4$ /group. (J) Quantitative PCR for $\beta$-arrestin 2 mRNA expression in $\mathrm{SAD}$ wild-type cardiomyocytes (referenced to expression in mouse brain cortex samples). No significant differences were found between groups. 


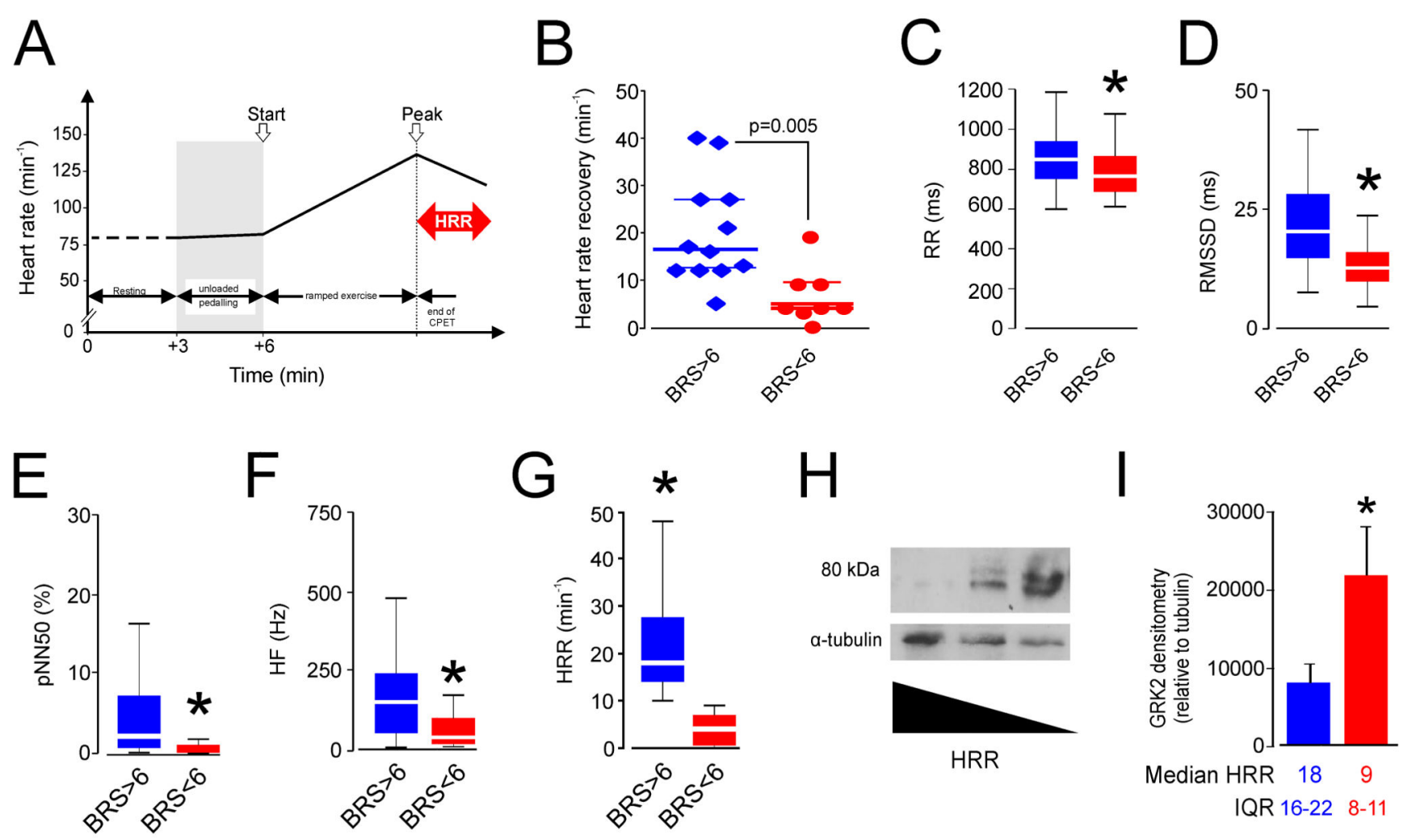

Figure 4.

Cardiopulmonary exercise performance to detect established preoperative baroreflex dysfunction. (A) Analysis of heart rate changes to determine heart rate recovery (HRR) as measure of parasympathetic cardiac activity in surgical patients undergoing preoperative cardiopulmonary exercise testing. (B) Heart rate recovery in surgical patients undergoing preoperative cardiopulmonary exercise testing, stratified by normal or low spontaneous baroreflex sensitivity. (C) RR-(pulse) interval in surgical patients, stratified by normal or low spontaneous baroreflex sensitivity. (D) Root mean square of the successive differences (RMSSD) in surgical patients, stratified by normal or low spontanenous baroreflex sensitivity. (E) pNN50 in surgical patients, stratified by normal or low spontanenous baroreflex sensitivity. Asterisk denotes $\mathrm{p} \unlhd \mathbf{0} .01$. (F) High frequency spectral analysis component of heart rate in surgical patients, stratified by normal or low spontanenous baroreflex sensitivity. $(\mathrm{G})$ Heart rate recovery profiles in surgical patients enrolled into COMPETE-C hemodynamic trial, obtained following preoperative cardiopulmonary exercise testing (stratified by normal or low spontaneous baroreflex sensitivity). $(\mathrm{H})$ Immunoblot for GRK2 protein expression in isolated mononuclear cells obtained from 3 consecutively recruited surgical patients undergoing preoperative cardiopulmonary exercise testing. (I) Group data for patient immunoblots performed in 10 separate subjects demonstrating each heart rate recovery phenotype (5/group). Median (25-75 ${ }^{\text {th }}$ centile) heart rate recovery values shown below the bars for patients with HRR threshold values stratified by original BRS data. ${ }^{*}$ Significant difference $(\mathrm{P}<0.05)$. 

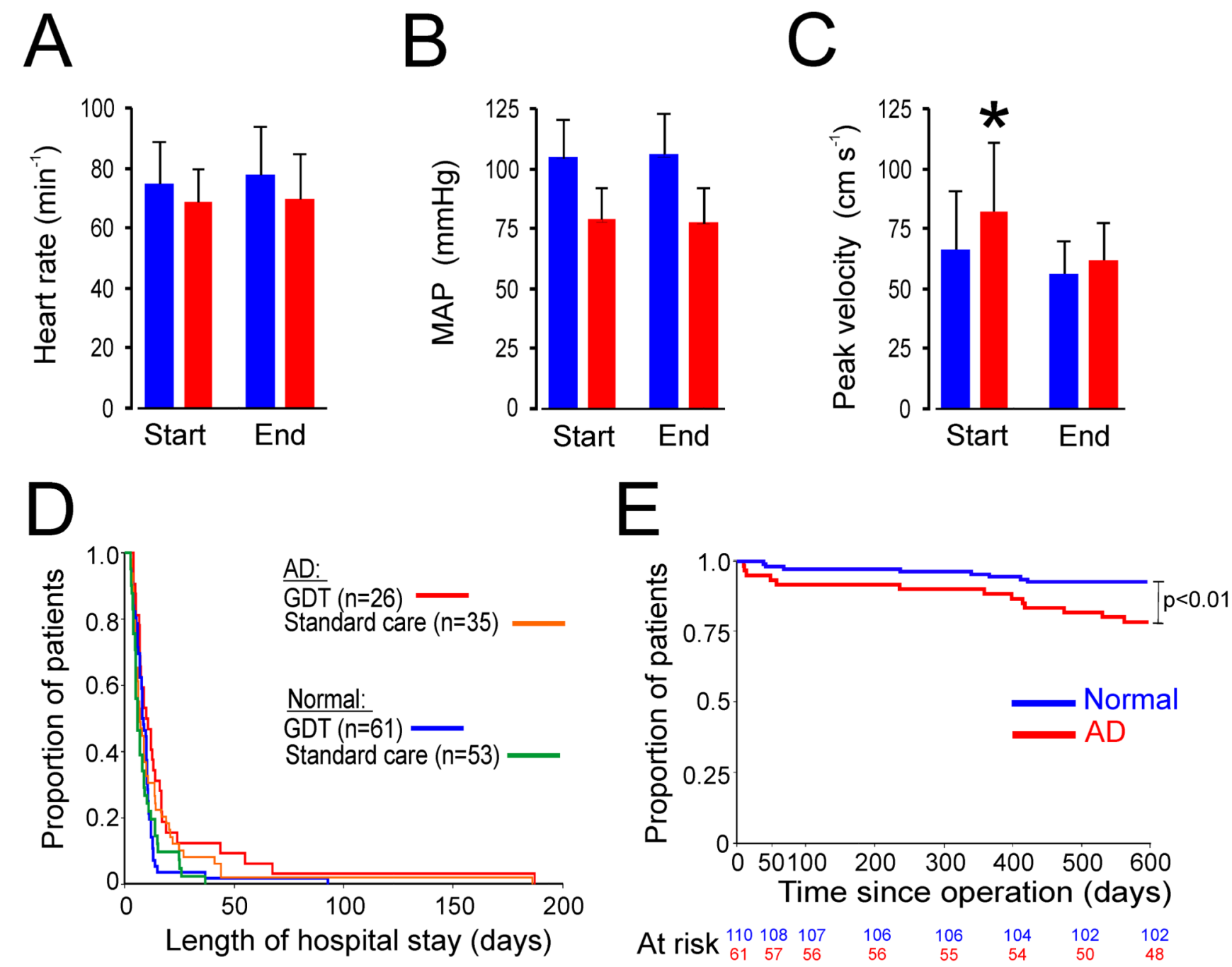

At risk $\begin{array}{cccccccc}110 & 108 & 107 & 106 & 106 & 104 & 102 & 102 \\ 61 & 57 & 56 & 56 & 55 & 54 & 50 & 48\end{array}$

Figure 5.

Perioperative cardiovascular performance in patients with autonomic dysfunction. (A) Heart rate during perioperative period; blue-normal HRR, red- HRR $<10 \mathrm{bpm}$ as defined in Figure 4A. (B) Mean arterial pressure during perioperative period. (C) Cardiac contractility (peak velocity) is impaired intraoperatively in patients with parasympathetic autonomic dysfunction defined by low HRR. (D) Prolonged hospital stay is observed in patients with preoperative parasympathetic autonomic dysfunction, regardless of intraoperative hemodynamic management (relative risk: 1.59 (95\% CI: 1.13-2.24); $\mathrm{p}=0.006$ ). GDT- patients randomized in COMPETE-C trial to goal directed therapy; AD: preoperative parasympathetic autonomic dysfunction. Values expressed as mean (SD). (E) Postoperative mortality is higher in patients detected to have parasympathetic autonomic dysfunction preoperatively after 600 days follow-up ( $<<0.001)$, by log rank test. 
Table 1.

Patients demographics for those who underwent CPET and for whom spontaneous baroreflex sensitivity was calculated preoperatively. Recruited as part of POM-O trial (ISRCTN76894700) patient cohort. Mean (SD) or n $(\%)$ shown.

\begin{tabular}{|c|c|c|}
\hline & BRS $>6 \mathrm{~ms} . \mathrm{mmHg}^{-1}(12)$ & BRS $<6 \mathrm{~ms} . \mathrm{mmHg}^{-1}(8)$ \\
\hline Age (y) & $64 \pm 7$ & $70 \pm 6$ \\
\hline Gender (male; \%) & $6(50 \%)$ & $6(75 \%)$ \\
\hline Body-mass index $\left(\mathrm{kg} \cdot \mathrm{m}^{-2}\right)$ & $26.5 \pm 6.1$ & $27.3 \pm 3.2$ \\
\hline Hemoglobin $\left(\mathrm{g} . \mathrm{l}^{-1}\right)$ & $122 \pm 13$ & $114 \pm 11$ \\
\hline Albumin $\left(\mathrm{g} \cdot \mathrm{L}^{-1}\right)$ & $41 \pm 6$ & $42 \pm 5$ \\
\hline Cardiac risk factor ${ }^{*}(\%)$ & $10(91 \%)$ & $8(100 \%)$ \\
\hline Anerobic threshold < $11 \mathrm{ml} \cdot \mathrm{kg} \cdot \mathrm{min}^{-1}$ & $9(75 \%)$ & $4(50 \%)$ \\
\hline Diabetes mellitus & $1(8 \%)$ & $0(0 \%)$ \\
\hline Malignancy & $8(75 \%)$ & $6(75 \%)$ \\
\hline
\end{tabular}

* Cardiac risk factors defined as meeting Revised Cardiac Risk Index criteria: aortic stenosis, established ischemic heart disease, cardiac failure, stroke/cerebrovascular event, diabetes mellitus, serum creatinine $>133 \mu \mathrm{mol} / \mathrm{L}$ 
Table 2.

COMPETE-C surgical patient cohort.

HRR could not be analysed from 4 patients as CPET was ended prematurely after the exercise test had finished, before heart rate recovery at 1 minute could be calculated. All values mean (SD) or n (\%).

\begin{tabular}{lll}
\hline & Normal & Autonomic dysfunction \\
\hline Number & 114 & 61 \\
Age yrs & $63(16)$ & $73(9)$ \\
Male (n, [\%]) & $66(58 \%)$ & $59(53 \%)$ \\
Body Mass Index, kg.m ${ }^{-2}$ & $27.3(3.8)$ & $29.4(4.9)$ \\
RCRI >2 & $12(11 \%)$ & $16(26 \%)$ \\
Malignancy & $77(68 \%)$ & $53(86 \%)$ \\
Perioperative Variables & & \\
General anaesthesia (GA) + neuraxial (n [\%]) & $71(62 \%)$ & $46(75 \%)$ \\
Open procedure (n (\%)) & $84(73 \%)$ & $51(69 \%)$ \\
Operative time (min) & $172(67)$ & $173(71)$ \\
Total Intraoperative fluid $\left(\mathrm{ml} \mathrm{kg}^{-1}\right)$ & $61(23)$ & $58(33)$ \\
Randomization to GDT $(\mathrm{n} ; \%)$ & $61(53 \%)$ & $26(43 \%)$ \\
Postoperative haemoglobin $\left(\mathrm{g} \mathrm{dl}^{-1}\right)$ & $10.9(1.8)$ & $10.4(1.5)$ \\
\hline
\end{tabular}

